\title{
"Postoperative Changes in Left Ventricular Systolic Function after Combined Mitral and Aortic Valve Replacement in Patients with Rheumatic Heart Disease" Sang-Mee An, Jae-Sik Nam et al
}

\author{
Fayyaz Hashmi ${ }^{1}$ \\ ${ }^{1}$ Johns Hopkins Medicine
}

May 10, 2021

\begin{abstract}
It is an elegant albeit limited study reporting effects of pre op LVEF on long term results in patients with RHD undergoing DVR. Study includes146 pqtients out of 201 who underwent DVR in the study period. Although all had some improvement immediate post op, those with preserved EF and smaller left ventricles regardless of type of prostheses used, surgical techniques ( partial or full Sub-valvular Apparatus Preservation), had more sustained improvement after 3-4 years than those with lower $\mathrm{EF}$ and more dilation. It can be partially explained by more prevalence of aortic insufficiency in patients with pre op lower EF $<50$ and dilation ( average LVESD $49 \mathrm{~mm}$ vs $32 \mathrm{~mm}$ in EF $>50$ ). There are myocardial factors which also play a part, those with abnormal LV function have more extensive loss of myofibrils either due to disproportion of mitochondria-to-myofibril ratio or myofibrillar degeneration exhibiting the extent RHD involves myocardium. Structural adaptation may not all be just a result of hemodynamic abnormalities in these patients (1). The recommendation that surgical intervention should occur before the LV starts to dilate or EF drops is well founded and would be impactful in the developing world, an estimated 250,000 deaths occur annually worldwide and 10.5 million disability adjusted life years due to RHD, mostly in young people.
\end{abstract}

\section{Hosted file}

Commentary on DVR in RHD, JOCS 2021.pdf available at https://authorea.com/users/412934/ articles/521432--postoperative-changes-in-left-ventricular-systolic-function-aftercombined-mitral-and-aortic-valve-replacement-in-patients-with-rheumatic-heart-diseasesang-mee-an-jae-sik-nam-et-al 